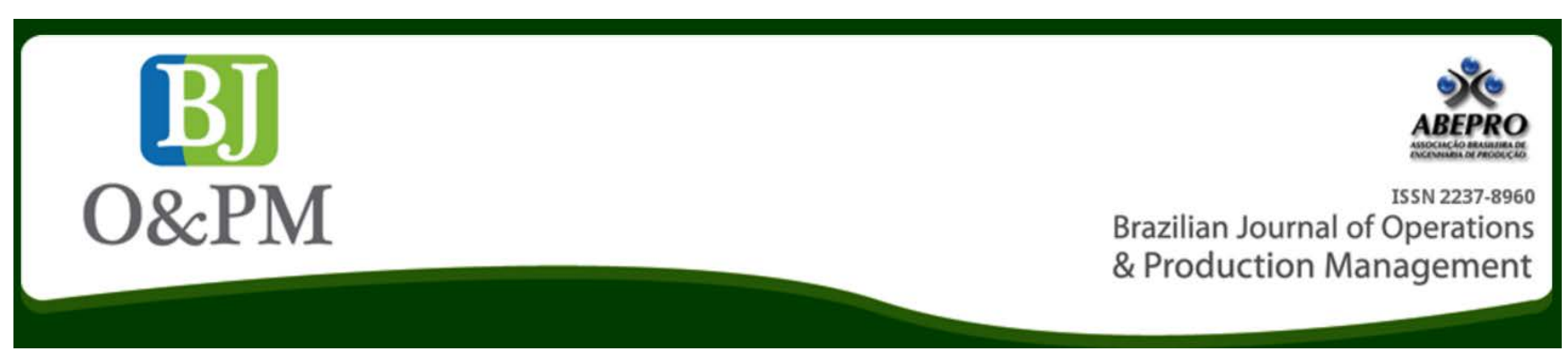

\title{
NEW HEURISTICS FOR THE NO-WAIT FLOWSHOP WITH SEQUENCE-DEPENDENT SETUP TIMES PROBLEM
}

\author{
Daniella Castro Araújo; ${ }^{a}$ Marcelo Seido Nagano ${ }^{a}$ \\ ${ }^{a}$ University of São Paulo (USP), São Paulo, SP, Brazil
}

Abstract

In this paper, we address the problem of scheduling jobs in a no-wait flow shop with sequence-dependent setup times with the objective of minimizing the make span and the total flow time. As this problem is well-known for being NPhard, we present two new constructive heuristics in order to obtain good approximate solutions for the problem in a short CPU time, named GAPH and QUARTS. GAPH is based on a structural property for minimizing make span and QUARTS breaks the problem in quartets in order to minimize the total flow time. Experimental results demonstrate the superiority of the proposed approaches over three of the best-know methods in the literature: BAH and BIH, from Bianco, Dell'Olmo and Giordani (1999) and TRIPS, by Brown, McGarvey and Ventura (2004).

Keywords: Heuristic, No-wait Flow shop, Sequence-Dependent Setup, Make span, Total flow time.

\section{INTRODUCTION}

The first systematic approach to scheduling problems was undertaken in the mid-1950s. Since then, thousands of papers on different scheduling problems have appeared in the literature. The majority of these papers assumed that the setup time is negligible or part of the job processing time. Treating setup times separately from processing times allows operations to be performed simultaneously and hence improves resource utilization. This is, in particular, important in modern production management systems such as just-in-time (JIT), optimized production technology (OPT), group technology (GT), cellular manufacturing (CM), and time-based competition (ALLAHVERDI et al., 2008). Another important area in scheduling arises in no-wait flow shop problems (NWFSP), where jobs have to be processed without interruption between consecutive machines. There are several industries where the no-wait flow shop problem applies including the metal, plastic, and chemical industries. As noted by Hall and Sriskandarajah (1996), the first of two main reasons for the occurrence of a no-wait or blocking production environment lies in the production technology itself. In some processes, for example, the temperature

ABEPRO

DOI: 10.14488/BJOPM.2015.v12.n2.a1 or other characteristics (such as viscosity) of the material require that each operation follow the previous one immediately. According to Bianco et al., (1999), flow shop no-wait scheduling problems are also motivated by concepts such as JIT and zero inventory in modern manufacturing systems.

A survey on NWFSP has been conducted by Hall and Sriskandarajah (1996), where several practical applications are shown. Allahverdi.et al., $(1999,2008)$ provided a comprehensive review of the literature on scheduling problems with setup times. The NWFSP with sequence dependent setup times with the objective of minimizing make span was first proposed by Bianco et al.,(1999). They showed how to reduce this problem to the asymmetric travelling salesman problem (ATSP) and presented two lower bounds and two heuristics, named $\mathrm{BAH}$ and $\mathrm{BIH}$. The computational results showed that BIH outperformed BAH in the solutions quality. Kumar et al.,(2000) considered a NWFSP that used lot-streaming to improve productivity. They developed a TSP formulation for the multi-product and continuous-sized case and proposed a heuristic to obtain an optimal sequence for integer-sized sublots. Allahverdi et Aldowaisan (2000) found optimal solutions 
for the $\frac{F 3}{S T_{s i}}$, no $-\frac{\text { wait }}{\sum C_{j}}$ problem, where the setup and processing times satisfy certain conditions, and presented five heuristics for the general problem. Later, Allahverdi et Aldowaisan (2001) considered the $\frac{F 2}{S T_{s d}}$, no $-\frac{\text { wait }}{\sum C_{j}}$ problem and presented five heuristics that used a repeated insertion technique. Stafford et Tseng (2002) proposed two mixed-integer linear programming (MILP) models to solve the $m$-machine NWFSP with sequence dependent setup times in order to minimize the make span. Aldowaisan et Allahverdi (2003) proposed six heuristics based on simulated annealing and genetic algorithms techniques for the $\frac{F_{m}}{n o}-\frac{\text { wait }}{C_{\max }}$ problem. The simulated annealing based heuristics performed better than the others. Fink et $V o \beta$ (2003) proposed three constructive heuristics and several meta-heuristics for the NWFSP with total flowtime as the criteria. Shyu et al., (2004) presented an ant colony optimization algorithm for the $\frac{F 2}{S T_{s i}}$, no $-\frac{\text { wait }}{\sum C_{j}}$ problem, and showed that their algorithm outperformed earlier heuristics.:- Brown et al., (2004) presented a non-polynomial time solution method and a heuristic named TRIPS for the NWFSP with sequence independent setup times, considering for the performance measures both the total flow time $\frac{F_{m}}{S T_{s d}}$ and make spanRuiz et al.,(2005) addressed the $\overline{C_{\max \square}}$ problem and proposed two genetic algorithms, named GA and HGA.- França et al., (2006) considered the same problem as- Bianco et al., (1999) and solved it by an evolutionary approach. Their genetic algorithm outperformed BIH. Ruiz et Allahverdi (2007a) presented a domination relation for the $\frac{F 4}{S T_{s i}}$,no- $\frac{\text { wait }}{\sum C_{j}}$ problem and proposed an iterated local search method and five heuristics for the same problem with $m$-machines. The results showed that three of their heuristics outperformed TRIPS and the ant colony algorithm of Shyu et al., (2004). Ruiz et Allahverdi (2007b) proposed seven heuristics and four genetic algorithms for the NWFSP with sequence independent setup times in order to minimize the maximum lateness. Their genetic algorithms outperformed the heuristics of Ruiz et Allahverdi (2007a). Grabowski et Pempera (2007) developed and compared five heuristcs for the $\frac{F_{m}}{n o}-\frac{\text { wait }}{C_{\max }}$ problem. In order to decrease the computational effort, they used multimoves. Ruiz et Stüzle (2008) presented two simple local search based iterated greedy algorithms for both $\frac{\frac{F_{m}}{S T_{s d}}}{\sum w_{j} T_{j}}$ and
$\frac{F_{m}}{C_{s d}}$
performed better than GA and HGA. Framinan et Nagano
(2008) studied the $\frac{F_{m}}{n o}-\frac{\text { wait }}{C_{\max }}$ problem and proposed a heuristic based on an analogy between the problem under consideration and the travelling salesman problem (TSP). Pan et al., (2008) presented a discrete particle swarm optimization (DPSO) to solve the NWFSP with both make span and total flow timecriteria. The results showed that the algorithm outperformed the heuristics of Grabowski et Pempera (2007) and Fink et Vo $\beta$ (2003). Yaurima et al.,(2009) proposed a genetic algorithm for the hybrid flow shop problem with unrelated machines, sequence dependent setup times, availability constraints and limited buffer, and introduced a crossover operator and stopping criterion to improve the solution quality. Eren (2010) proposed an integer programming model to solve the flow shop problem with sequence dependent setup times. The objective function was the weighted sum of total completion time and the make span. The model could solve problems up to six machines and eighteen jobs. Wang (2010) considered the NWFSP with maximum lateness criterion and developed properties to reduce the time to evaluate a candidate in a tabu search approach. Framinan et al., (2010) addressed the $\frac{F_{m}}{n o}-\frac{\text { wait }}{\sum C_{j}}$ based on an analogy with the two-machine probtic based on an analogy with the two-machine problem. The computational results showed that the heuristic outperformed existing ones regarding the solution quality.

In this paper, we consider the problem of scheduling a no-wait flow shop problem with sequence dependent setup times $\left(\frac{F_{m}}{S T_{s d}}\right.$, no - wait $)$, which consists of a set $\mathbf{J}=\left\{j_{1}, j_{2}, j_{3}, \ldots, j_{n}\right\}$ of $n$ jobs which are to be processed on a set $M=\left\{m_{1}, m_{2}, m_{3}, \ldots, m_{m}\right\}$ of $m$ dedicated machines, each one being able to process only one job at a time. Job $i_{i}$ consists of $m$ operations $o p_{k i}, o p_{k+1 i}, \ldots, o p_{m i}$, to be executed in this order, where operation $O p_{k i}$ must be executed on machine $k$, with $p_{k i}$ processing time, immediately before operation $O p_{k+1 i}$. There is a sequence dependent setup time $s_{i j}^{k}$ between operations $o p_{k i}$ and $o p_{k j}$ in machine $k$. We propose two new heuristic methods for the problem, GAPH and QUARTS, in order to minimize the make span and the total flow time, respectively. GAPH is based on a property of the scheduling problem that provides 
the time break between the beginning of job $j_{[i+1]}$ and the beginning of job $j_{[j]}$ at machine $k$, where, $j_{[j]}$ is the job of J occupying position $i$ in $\sigma$. QUARTS breaks the problem in quartets, reducing the required computational effort to find the solution.

\section{EXISTING CONSTRUCTIVE HEURISTICS FOR THE PROBLEM}

In this section, we review the main contributions to the problem regarding constructive methods. More specifically, we explain in detail the constructive heuristics BAH and BIH, from Bianco et al., (1999) and TRIPS, from Brown et al.,(2004). BAH and BIH were made for the no-wait flow shopwith sequence-dependent setup times in order to minimize the make span, and were adapted in this work to also minimize the total flow time. TRIPS was made for the no-wait flow shop with sequence-independent setup times, to minimize both the make span and total flow time, and was adapted in this work to the no-wait flow shopwith sequence-dependent setup times.

\section{BAH}

BAH algorithm finds a feasible sequence in $n$ iterations. At each iteration, given a partial sequence of the scheduled jobs computed in the previous iteration, the algorithm examines a set of candidates of the unscheduled jobs, and appends a candidate job to a partial sequence minimizing the time when the shop is ready to process an unscheduled job.

The pseudo-code of the heuristic is as follows:

Given a set $\mathbf{I}=\left\{j_{1}, j_{2}, j_{3}, \ldots, j_{n}\right\}$ of $n$ jobs, let $\sigma$ be the set of programmed jobs and $U$ be the set of non-programmed jobs.

Step 1: $\mathrm{U} \leftarrow \mathbf{J} ; \sigma \leftarrow \emptyset ;$

Step 2: While $\mathrm{U} \neq \mathbf{]}$, do:

Step 2.1: Choose the job $j_{i} \in U$ to be added at the end of the sequence $\sigma$, such that the makespan/flowtime is minimum;

Step 2.2: Add job $j_{i}$ to the end of the sequence $\sigma$;

Step 2.3: $\mathrm{U} \leftarrow \mathrm{U}-j_{i}$.

\section{BIH}

The BIH algorithm also finds a sequence of $n$ jobs on $n$ iterations. But in this algorithm, at each iteration it considers a sequence of a subset of jobs, and finds the best sequence obtained inserting an unscheduled job in any position of the given sequence.

A more detailed description of the heuristic is as follows:

Given a set $\mathbf{J}=\left\{j_{1}, j_{2}, j_{3}, \ldots, j_{n}\right\}$ of $n$ jobs, let $\sigma$ be the set of programmed jobs, $U$ be the set of non-programmed jobs and $h$ the relative insertion position.

Step 1: $\mathrm{U} \leftarrow \mathbf{J} ; \sigma \square \leftarrow$

Step 2: While $\mathrm{U} \neq[$, do:

Step 2.1: Choose the job $j_{i} \in U$ which can be inserted in the sequence $\sigma$, such that the make span/flow time is minimum. Let $h$ be the relative insertion position;

Step 2.2: Insert job $j_{i}$ at position $h$ in the sequence $\sigma$;

Step 2.3: $\mathrm{U} \leftarrow \mathrm{U}-j_{i}$.

\section{TRIPS}

TRIPS examines all possible three-job combinations from the set of unscheduled jobs $\mathbf{U}$ and chooses the sequence $\left\{j_{w}, j_{x}, j_{y}\right\}$ that minimizes the three-job objective. Then, assigns job $\mathbf{j}_{\mathbf{w}}$ to the last empty position in the sequence $\sigma$ and removes $\mathbf{j}_{\mathbf{w}}$ from $U$. The heuristic repeats the process, assigning one more job to $\sigma$ for each set of triplets examined until only three jobs are left. Then, it selects the optimal sequence for these jobs and places them in the final positions of heuristic sequence $\sigma$.

The pseudo-code of the heuristic is as follows:

Given a set $\mathbf{J}=\left\{j_{1}, j_{2}, j_{3}, \ldots, j_{n}\right\}$ of $n$ jobs, let $\sigma$ be the set of programmed jobs and $U$ be the set of non-programmed jobs.

Step 1: $\mathrm{U} \leftarrow \mathbf{J} ; \sigma \leftarrow \Xi ; h \leftarrow \mathbf{0} ;$

Step 2: While $h<n-2$, do:

Step 2.1: Given that the first $h$ jobs are assigned in sequence $\sigma$, compare all ordered triplets of jobs from $U$;

Step 2.2: Choose the triplet $\left\{\mathbf{j}_{w}, j_{x}, j_{y}\right\}$ such that the make span/flow time is minimized for jobs $\left\{\mathbf{j}_{w}, \mathbf{j}_{x}, \mathbf{j}_{y}\right\}$ in positions $h+1, h+2, h+3$, respectively, of sequence $\sigma$.

Step 2.3: Place $\mathbf{j}_{w}$ in position $h+1$ of $\sigma$;

Step 2.4: $h \leftarrow h+1 ; \mathrm{U} \leftarrow \mathrm{U}-\mathrm{j}_{\mathrm{W}}$;

Step 3: Assign $\dot{j}_{\mathbf{x}}$ and $\mathbf{j}_{\mathrm{y}}$ to the last two positions, respectively, of $\sigma$. 
A STRUCTURAL PROPERTY FOR THE NEW HEURISTIC FOR MAKESPAN

Given a sequence $\sigma$ of $I, j_{[j]}$ is the job of $I$ occupying position $i$ in $\sigma$. The time break between the beginning of job $j_{[i+1]}$ and the beginning of job $j_{[j]}$ at machine $k$ is $\Delta t_{[j][i+1]}^{k}$ , calculated as follows:
Defining $G A P_{[i][i+1]}^{k}$ as the time break between the end of job $j_{[j]}$ and the beginning of job $j_{[i+1]}$ at machine $k$, it can be calculated as follows:

$$
G A P_{[i][i+1]}^{k}=\Delta t_{[\mathbf{i}[\mathbf{i}+\mathbf{1}]}^{k}-p_{k[\mathbf{i}]}
$$

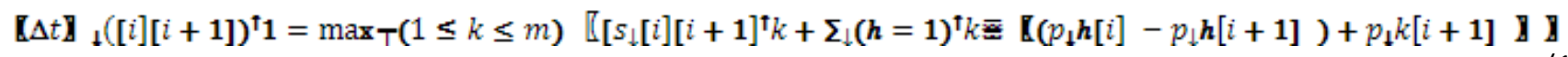

$$
\Delta t_{[i][i+1]}^{k}=\Delta t_{[i][i+1]}^{1}+\sum_{h=1}^{k-1}\left(p_{h[i+1]}-p_{h[i]}\right)
$$

The GAP of the first job in the sequence on machine $k$ is defined by expression (4):

$$
G A P_{[0][1]}^{k}=\sum_{h=1}^{k-1} p_{k[\mathbf{b}]}
$$

Figure 1 shows the time break between the end of job $\bar{j}_{\mathbf{2}}$ and the beginning of job $j_{3}$ on machine $2\left(G A P_{[2][3]}^{2}\right)$.

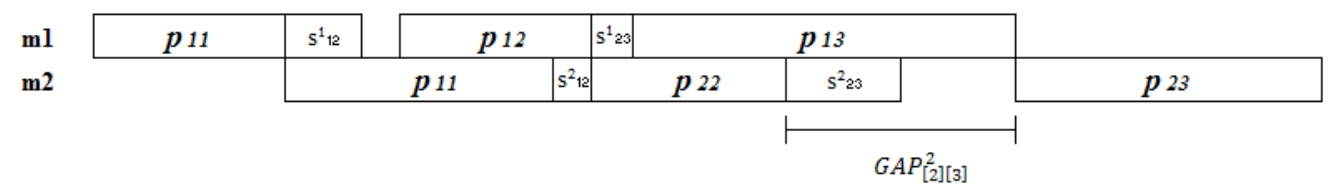

Figure 1 - Example of the GAP calculation

\section{THE NEW HEURISTIC FOR MAKESPAN}

The new heuristic proposed in this paper will be called GAPH - Gap Heuristic. The pseudo-code of the algorithm is given next:

Given a set $\mathbf{J}=\left\{j_{1}, j_{2}, j_{2}, \ldots, j_{n}\right\}$ of $n$ jobs, let $U$ be the set of non-programmed jobs and $\sigma_{\mathrm{x}}=\left(j_{[1]}, j_{[2]}, \ldots, j_{[n]}\right)$ be the sequence of $n$ jobs scheduled, where $x=\{1,2,3,4\}$. Calculate the $G A P_{[i][j]}^{k}$ of each job $i=1, \ldots, n$ to each job $j$ $=1, \ldots, n$ at all $m$ machines.

Step 1: $U \leftarrow \mathrm{J} ; \sigma_{1} \leftarrow \varnothing$;

Step 2: While $U \neq \varnothing$, do:

Step 2.1: Calculate the total cost* on the last machine for all possible insertions of each job $j_{i} \in U$ in the sequence $\sigma_{1}$. Let $h$ be the relative insertion position;

Step 2.2: Choose the job $j_{i}$ that gives the lower total cost at position $h$;

Step 2.3: Insert job $j_{i}$ at position $h$ of the sequence $\sigma_{1}$;
Step 2.4: $U \leftarrow U-j_{i}$;

Step 3: $U \leftarrow \mathrm{J} ; \sigma_{2} \leftarrow \varnothing$

Step 4: While $U \neq \varnothing$, do:

Step 4.1: Calculate the total $G A P^{* *}$ for all possible insertions of each job $j_{i} \in U$ in the sequence $\sigma_{2}$. Let $h$ be the relative insertion position;

Step 4.2: Choose the job $\dot{j}_{i}$ that gives the lower total GAP at position $h$;

Step 4.3: Insert job $j_{i}$ at position $h$ of the sequence $\sigma_{2}$;

Step 4.4: $U \leftarrow U-j_{i}$;

Step 5: $U \leftarrow \mathrm{J} ; \sigma_{3} \leftarrow \varnothing$;

Step 6: While $U \neq \varnothing$, do:

Step 6.1: Calculate the sum of the GAPs on the last machine for all possible insertions of each job $j_{i} \in U$ in the sequence $\sigma_{3}$. Let $h$ be the relative insertion position;

Step 6.2: Choose the job $j_{i}$ that gives the lower sum of 
the GAPs on the last machine at position $h$;

Step 6.3: Insert job $j_{i}$ at position $h$ of the sequence $\sigma_{3}$;

Step 6.4: $U \leftarrow U-j_{i}$;

Step 7: $U \leftarrow \mathrm{J} ; \sigma_{4} \leftarrow \varnothing$;

Step 8: While $U \neq \varnothing$, do:

Step 8.1: Calculate, for all possible insertions of each job $j_{i} \in U$ in the sequence $\sigma_{4}$, the sum of the GAPs with the processing time of the job on the last machine. Let $h$ be the relative insertion position;

Step 8.2: Choose the job $j_{i} \in U j_{i}$ that gives the lower sum of the GAPs with the processing time of the job on the last machine at position $h$;
Step 8.3: Insert job $j_{i}$ at position $h$ of the sequence $\sigma_{4}$.

Step 8.4: $U \leftarrow U-j_{i}$;

Step 9: Choose, among the sequences $\left\{\sigma_{1}, \sigma_{2}, \sigma_{3}, \sigma_{4}\right\}$ , the one with the lower make span.

*The total cost on a $k$ machine is defined as the scheduling total time on this machine. Thus, the total cost encompasses the sum of the GAPs on machine $k$ with the scheduled operations processing times on that machine. Note that the total cost on the last machine is equivalent to the (see Figure 2).

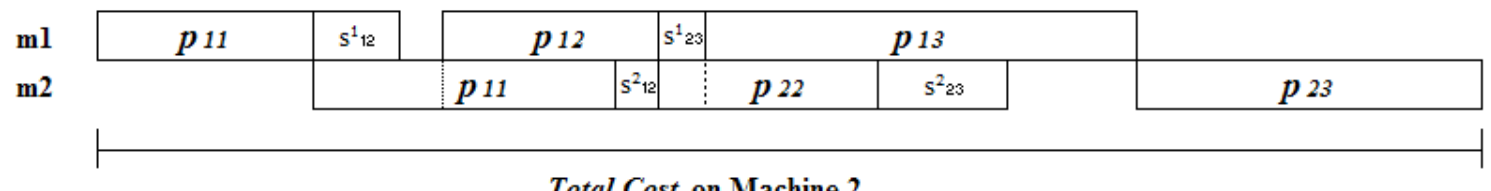

Total Cost on Machine 2

Figure 2 - Example of the total cost

**The total GAP is the sum of all GAPs in all machines.

The sum of the GAPs on the last machine is:

In Figure 3 , the total $G A P$ is:

$G A P_{[1][2]}^{1}+G A P_{[2][3]}^{1}+G A P_{[0][1]}^{2}+G A P_{[1][2]}^{2}+G A P_{[2][3]}^{2}$

$$
G A P_{[0][1]}^{2}+G A P_{[1][2]}^{2}+G A P_{[2][3]}^{2} \text {. }
$$

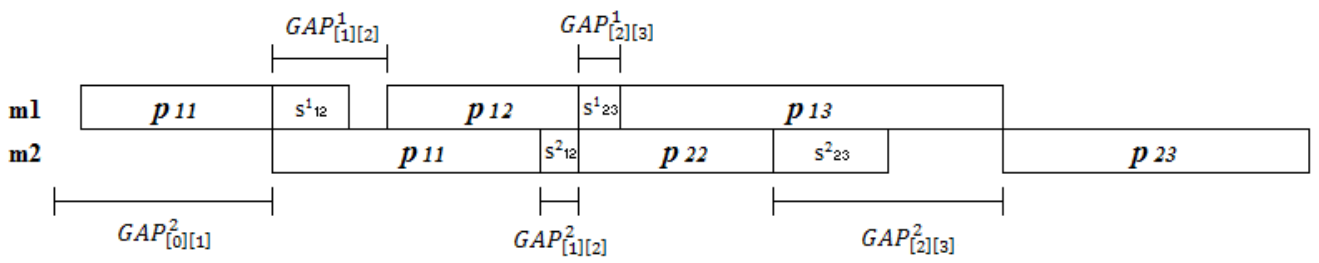

Figure 3 - Example of all the GAPs in the scheduling

The sum of the GAPs with the processing time of the job to be inserted on the last machine is: $G A P_{[0][1]}^{2}+G A P_{[1][2]}^{2}+G A P_{[2][3]}^{2}+p_{23}$.
Figure 4 shows how the method works for each pair of steps $(1-2 ; 3-4 ; 5-6 ; 7-8)$. In the example, jobs $\bar{h}_{\mathbf{1}}$ and $\bar{j}_{\mathbf{2}}$ were already scheduled $\left(\sigma=\left(j_{1}, j_{2}\right)\right.$ ) and job $j_{3}$ is scheduled on each possible position of the sequence $(h)$. 
Jobs scheduled:

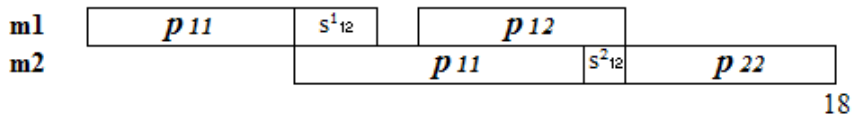

1. Job $\mathrm{j}_{3}$ is inserted in position $h=1$ :

\begin{tabular}{|c|c|c|c|c|c|c|}
\hline$p_{13}$ & $\mathrm{~s}^{1}{ }_{31}$ & $p_{11}$ & $\mathrm{~s}^{1}{ }_{12}$ & & & \\
\hline $\mathrm{m} 2$ & $p 23$ & $\mathrm{~s}^{2}{ }_{31}$ & & $p_{21}$ & $s^{2}+12$ & $p 22$ \\
\hline
\end{tabular}

2. Job $\mathrm{j}_{3}$ is inserted in position $h=2$ :

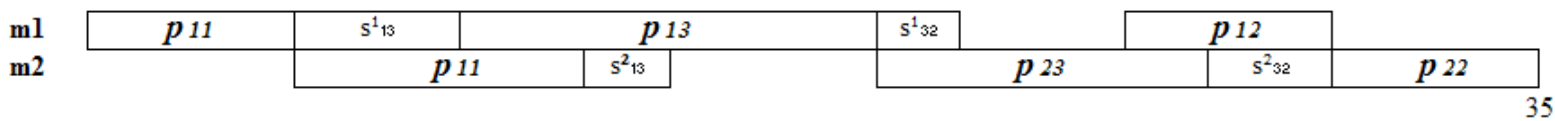

3. Job $\mathrm{j}_{3}$ is inserted in position $h=3$ :

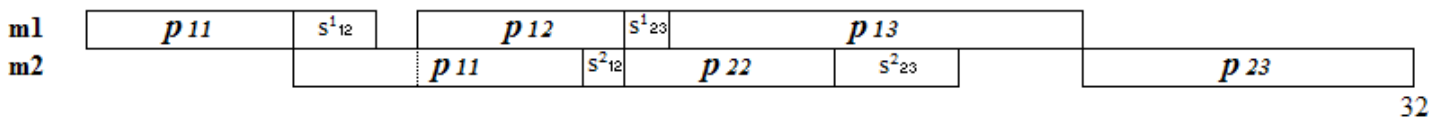

Figure 4 - Numerical example of how the method works

For example, if the method were on the second step, the sequence chosen would be the third one, that gives the lower total cost on the last machine.

\section{DESCRIPTION OF THE NEW HEURISTIC FOR THE TOTAL FLOWTIME}

Brown et al., (2004) presented the TRIPS heuristic detailed above. TRIPS breaks the problem in triplets, reducing the required computational effort to find the solution. We made some modifications in this heuristic in order to improve its results. The main difference of the proposed heuristic is that it breaks the problem in quartets, instead of triplets. The new heuristic will be called QUARTS.

In order to choose the two first jobs, QUARTS examines all possible four-job combinations from the set of jobs $J$ and chooses the sequence $\left\{\mathbf{j}_{w}, \mathbf{j}_{\mathrm{x}}, \mathbf{j}_{\mathrm{y}}, \mathrm{j}_{\mathrm{z}}\right\}$ that minimizes the total flow time. Then assigns jobs $\mathbf{j}_{\mathrm{w}}$ and $\mathbf{j}_{\mathrm{x}}$ to the first and second positions of $\sigma$, respectively, and removes them from the set of non-scheduled jobs. In the next iteration, the job scheduled in the second position of $\sigma$ will be the first

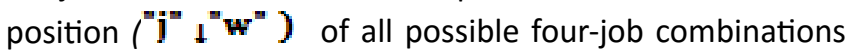
$\left\{\mathbf{j}_{\mathrm{w}}, \mathbf{j}_{\mathrm{x}}, \mathbf{j}_{\mathrm{y}}, \mathbf{j}_{\mathrm{z}}\right\}$. The job in the second position $\left(\mathbf{j}_{x}\right)$ of the quartet with the lowest flow time is then scheduled in the next empty position of $\sigma$ and is removed from the set of non-scheduled jobs. Repeating this process, QUARTS assigns one job in $\sigma$ at each iteration, and the job scheduled in the previous iteration is always the first one of the quartets of the current iterations, until only three jobs are left. Select the optimal sequence for these jobs and place them in the final positions of heuristic sequence $\sigma$. Then, QUARTS tries to improve the solution found through a neighborhood insertion and a neighborhood permutation search procedures, described as follows:

- Neighborhood Insertion Search:

Insert each job $j_{i}$ in the sequence $\sigma$ at the $(n-1)^{2}$ possible positions, being $n$ the number of jobs. Choose the insertion that obtains the lower total flowtime.

- Neighborhood Permutation Search:

This search exchanges pairs of tasks in the sequence $\sigma$, at the $\frac{n(n-1)}{2}$ possible positions. Choose the insertion that obtains the lower total flowtime.

The pseudo-code of the algorithm is given next:

Given a set $\mathbf{J}=\left\{j_{1}, j_{2}, j_{3}, \ldots, j_{n}\right\}$ of $n$ jobs, let $U$ be the set of non-programmed jobs and $\sigma=\left(j_{[1]}, j_{[2]}, \ldots, j_{[n]}\right)_{\text {be }}$ the sequence of $n$ jobs scheduled. 
Step 1: $U \leftarrow \mathrm{J} ;{ }^{\sigma, \sigma^{\prime}, \sigma^{\prime \prime} \leftarrow \text { ] }} ; h^{\leftarrow \mathbf{1}}$.

Step 2:

Step 2.1: Calculate the total flow time for all possible fourjob combination of $U$;

Step 2.2: Choose the quartet $\left\{\mathbf{j}_{w}, \mathbf{j}_{\mathrm{x}}, \mathbf{j}_{\mathrm{y}}, \mathbf{j}_{\mathbf{z}}\right\}$ that gives the lower flow time and assign $\mathbf{j}_{\mathbf{w}}$ and $\mathbf{j}_{\mathbf{x}}$ in positions 1 and 2 of $\sigma$, respectively;

Step 2.3: $\mathrm{h} \leftarrow \mathrm{h}+2 ; \mathbf{U} \leftarrow \mathbf{U}-\mathbf{j}_{\mathrm{w}} ; \mathbf{U} \leftarrow \mathbf{U}-\mathbf{j}_{\mathbf{x}}$.

Step 3: While $\mathrm{h}<\mathrm{n}-2$, do:

Step 3.1: $\mathbf{j}_{w} \leftarrow \mathbf{j}[h-1]$;

Step 3.2: Calculate the flow time for all quartets $\left\{\mathbf{j}_{w}, \mathrm{j}_{\mathrm{x}}, \mathrm{j}_{\mathrm{y}}, \mathrm{j}_{\mathrm{z}}\right\}$ of $U$;

Step 3.3: Choose the quartet that gives the lower flow time and assign $\mathbf{j}_{\mathbf{w}}$ to the position $h$ of $\sigma$;

Step 3.4: $h \leftarrow h+1 ; \mathbf{U} \leftarrow \mathbf{U}-\mathbf{j}_{x} ;$

Step 4: Assign $\mathrm{j}_{\mathrm{y}}$ and $\mathrm{j}_{z}$ to the last positions, respectively, of $\sigma$. Calculate the total flow time.

Step 4.1: Calculate the total GAP** for all possible insertions of each job $j_{i} \in U$ in the sequence $\sigma_{2}$. Let $h$ be the relative insertion position;

Step 4.2: Choose the job $j_{i}$ that gives the lower total GAP at position $h$;

Step 4.3: Insert job $j_{i}$ at position $h$ of the sequence $\sigma_{2}$;

Step 4.4: $U \leftarrow U-j_{i}$;

Step 5: Neighborhood insertion search: Considering all insertion neighborhood of sequence $\sigma$, determine the sequence $\sigma^{\prime}$ that gives the lower total flow time. If the total flow time of the sequence $\sigma^{\prime}$ is lower than the sequence $\sigma$, then $\sigma^{\leftarrow} \sigma^{\prime}$.

Step 6: Neighborhood permutation search: Considering all insertion neighborhood of sequence $\sigma$, determine the sequence $\sigma^{\prime \prime}$ that gives the lower total flow time. If the total flow time of the sequence $\sigma^{\prime \prime}$ is lower than the sequence $\sigma$, then $\sigma^{\leftarrow} \sigma^{\prime \prime}$.

Figures 5, 6 and 7 give an example of the method, in a problem with 5 jobs and 3 machines.

First of all, QUARTS calculates the flow time of all quartets $\left\{\mathbf{j}_{w}, \mathbf{j}_{\mathrm{x}}, \mathbf{j}_{\mathrm{y}}, \mathbf{j}_{z}\right\}$. Then, it picks up the one with the lowest flow time. Figure 5 shows the selected quartet $\left(\left\{\mathbf{j}_{3}, \mathbf{j}_{5}, \mathbf{j}_{1}, \mathbf{j}_{2}\right\}\right)$.

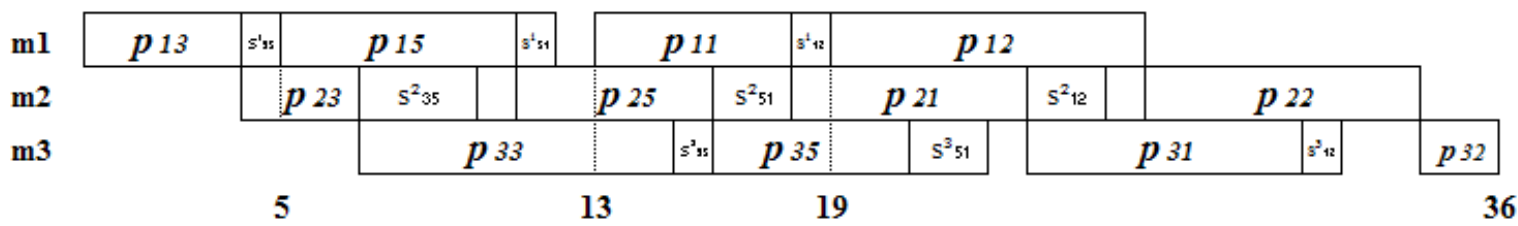

Figure 5 - First selected quartet

The first and second jobs of the quartet $\left\{\mathrm{j}_{3}, \mathrm{j}_{5}, \mathrm{j}_{1}, \mathrm{j}_{2}\right\}$ are then programmed in the first and second positions of the set of programmed jobs $(\sigma)$, and are removed from the set of non-programmed jobs $(U)$. In the next iteration, job $\mathbf{i}_{5}$ will be the first position of all quartets.
After calculating the flow time of all quartets $\left\{\mathrm{j}_{5}, \mathrm{j}_{\mathrm{x}}, \mathrm{j}_{\mathrm{y}}, \mathrm{j}_{\mathrm{z}}\right\}$ , QUARTS picks up the one with the lowest flow time. Figure 6 shows the selected quartet $\left(\left\{\mathbf{j}_{5}, \mathbf{j}_{1}, j_{4}, \mathbf{j}_{2}\right\}\right)$.

\begin{tabular}{|c|c|c|c|c|c|c|c|c|c|c|c|}
\hline \multirow{3}{*}{$\begin{array}{l}\mathrm{ml} \\
\mathrm{m} 2\end{array}$} & \multirow[t]{2}{*}{$3^{1} s_{1}$} & \multicolumn{2}{|c|}{$p_{11}$} & \multirow{2}{*}{\begin{tabular}{l|l|}
$s^{1}{ }_{14}$ & \\
& $p 21$ \\
\end{tabular}} & \multicolumn{2}{|c|}{$p_{14}$} & \multirow[t]{2}{*}{$\mathrm{S}^{1}{ }_{42}$} & \multicolumn{2}{|l|}{$p_{12}$} & \multirow{2}{*}{\multicolumn{2}{|c|}{$p_{22}$}} \\
\hline & & p 25 & $\mathrm{~s}^{2} 51$ & & & $\mathrm{~s}^{2} 14$ & & $p_{24}$ & $\mathrm{~s}^{2} 42$ & & \\
\hline & & & & & $\mathrm{s}^{3} 51$ & & $p_{31}$ & $\mathrm{~s}^{3} 14$ & $p_{34}$ & $s^{3} 42$ & $p 32$ \\
\hline
\end{tabular}

Figure 6 - Second selected quartet 
As there are only three jobs to be programmed, all of the non-programmed jobs of this quartet will be programmed in the last positions of $\sigma$. Thus, $\mathbf{j}_{1}, \quad j_{\mathbf{4}} e j_{2}$ will be programmed in the third, fourth and fifth positionsof $\sigma$ , respectively, and removed from $U$. So, the final sequence will be $\left\{\mathbf{j}_{3}, \mathbf{j}_{5}, \mathbf{j}_{1}, j_{4}, j_{2}\right\}$ (Figure 7).

\begin{tabular}{|c|c|c|c|c|c|c|c|c|c|c|c|c|c|c|}
\hline \multirow[t]{2}{*}{$p_{13}$} & \multirow[t]{2}{*}{$s^{\prime} s$} & \multicolumn{2}{|c|}{$p_{15}$} & \multirow[t]{2}{*}{$\mathrm{s}^{1} \mathrm{st}_{1}$} & \multicolumn{2}{|l|}{$p_{11}$} & $\mathrm{~s}^{1} 14$ & \multicolumn{2}{|c|}{$p_{14}$} & $\mathrm{~S}^{1} 42$ & \multicolumn{2}{|l|}{$p_{12}$} & \multirow{2}{*}{\multicolumn{2}{|c|}{$p 22$}} \\
\hline & & $p_{23}$ & $\mathrm{~s}^{2} 35$ & & $p_{25}$ & $s^{2} 51$ & & & $\mathrm{~s}^{2} 14$ & & p 24 & $\mathrm{~S}^{2} 42$ & & \\
\hline & & & & & $s^{2} y$ & $p$ & & $s^{3} 51$ & & 31 & $\mathrm{~s}^{3} 14$ & & $\mathrm{~s}^{3} 42$ & p32 \\
\hline
\end{tabular}

Figure 7 - Final sequence

Then, the heuristic tries to improve the result with the neighborhood insertion and permutation searches.

\section{COMPUTATIONAL EXPERIENCE}

The computational experience was carried out in two parts. In part I, we tested GAPH, BIH, BAH and TRIPS with the make span criteria. In part II, we tested QUARTS, BIH, BAH and TRIPS with the total flow time criteria. As stated, TRIPS heuristic was adapted to the no-wait flow time with sequence-dependent setup times problem, and $\mathrm{BAH}$ and $\mathrm{BIH}$ were adapted in order to also minimize the total flow time.

The heuristics were tested in the well-known tested of Taillard (1993). This tested contains twelve sets for a given combination of $j$ obs and machines, i.e., $n\{20,50,100,200,500\}$ and $m\{5,10,20\}$. We performed four experiments, one for each of the four different sequence-dependent Taillardbased instance sets from- (Ruiz et al. ,(2005). The tests contain four different processing times to sequencedependent setup times ratios. For example, the instance set SSD-10 is composed of one hundred twenty instances where the processing times are those of Taillard's benchmark and where the sequence-dependent setup times are $10 \%$ of the processing times. In the instance set SSD-50, the setup times are $50 \%$ of the processing times and the instance sets SSD100 and SSD-125 have setup times that are $100 \%$ and $125 \%$ of the processing times respectively. So for example, if the processing times in Taillard's instances are generated from a uniform distribution in the range $[1 ; 99]$, in the SSD-10 instance set the setup times are uniformly distributed in the range $[1 ; 9]([1 ; 49],[1 ; 99]$ and $[1 ; 124]$ for the instance sets SSD-50, SSD-100 and SSD-125 respectively). Thus, we have four problem sets and a total of 480 different instances. The five hundred job instance was rather large and we chose to solve only the first one hundred ten instances (up to two hundred jobs and twenty machines).

The instances in the tested have been solved by the selected heuristics (coded in Python). Part I was solved in a computer with a Pentium IV $3.00 \mathrm{GHz}$ processor and 512MB RAM, and Part II in a computer with a Intel Core 2 Duo $2.20 \mathrm{GHz} / 2.20 \mathrm{GHz}$ processor and 4.00GB RAM.
Tables 1 and 2 summarize the result obtained for the make span criteria and Tables three and-four for the total flow time criteria in terms of the success percentage, the average relative percentage deviation (ARPD), and the average CPU time.

The success rate is defined by the ratio between the number of problems for which a particular method was the best solution and the total number of problems solved. Therefore, when two methods get the best solution for the same problem, their percentages of success are both improved.

The ARPD consists of averaging the RPD over a number of instances with the same number of jobs. We have grouped the results for a given number of jobs and different machines, as the number of machines had almost no influence in the results. For a given objective function $f$, the RPD obtained by a heuristic $\mathrm{H}$ on a given instance is computed as follows:

$$
R P D(H)=\frac{f(H)-f^{*}}{f^{*}} \cdot 100
$$

where: method $h$;

$f(H)$ is the makespan/total flowtime computed by

$f^{*}$ is the best makespan/total flowtime computed by the methods.

As we can see from the results in Tables 1 and 2, the proposed heuristic obtains better results than the rest of the constructive heuristics. Over all configurations, the maximal ARPD from the best solution found was $0.14 \%$ for GAPH (when TRIPS found the best solution) and $2.48 \%$ for BIH. The maximal ARPD for BAH was $15.87 \%$ and $9.27 \%$ for TRIPS. All success rates for BAH were zero, and TRIPS only got the best solution once. So, we can conclude that BAH and TRIPS are not competitive with the other heuristics tested. 
Brazilian Journal of Operations \& Production Management

Volume 12, Número 2, 2015, pp. 180-194

DOI: 10.14488/BJOPM.2015.v12.n2.a1

Table 1 - Comparison of results in Taillard's testbed SSD-10 and SSD-50 (Makespan)

\begin{tabular}{|c|c|c|c|c|c|c|c|c|}
\hline \multirow[b]{2}{*}{$n \times m$} & \multicolumn{4}{|c|}{ SSD-10 } & \multicolumn{4}{|c|}{ SSD-50 } \\
\hline & BAH & BIH & TRIPS & GAPH & BAH & BIH & TRIPS & GAPH \\
\hline \multirow{3}{*}{$20 \times 5$} & $0.00 *$ & 60 & 0 & 100 & 0 & 60 & 0 & 100 \\
\hline & $15.54 * *$ & 1.83 & 9.27 & 0 & 13.13 & 1.27 & 7.23 & 0 \\
\hline & $0.03^{* * *}$ & 0.11 & 0.34 & 0.27 & 0.03 & 0.11 & 0.34 & 0.27 \\
\hline \multirow{3}{*}{$20 \times 10$} & 0 & 50 & 0 & 100 & 0 & 70 & 0 & 100 \\
\hline & 15.87 & 2.48 & 8.23 & 0 & 14.21 & 1.59 & 8.09 & 0 \\
\hline & 0.06 & 0.15 & 0.37 & 0.31 & 0.06 & 0.15 & 0.36 & 0.31 \\
\hline \multirow{3}{*}{$20 \times 20$} & 0 & 60 & 0 & 100 & 0 & 70 & 0 & 100 \\
\hline & 13.42 & 0.42 & 8.82 & 0 & 13.21 & 0.88 & 8.37 & 0 \\
\hline & 0.18 & 0.26 & 0.43 & 0.43 & 0.17 & 0.26 & 0.42 & 0.43 \\
\hline \multirow{3}{*}{ Average } & 0 & 56.67 & 0 & 100 & 0 & 66.67 & 0 & 100 \\
\hline & 14.94 & 1.58 & 8.77 & 0 & 13.51 & 1.25 & 7.9 & 0 \\
\hline & 0.09 & 0.17 & 0.38 & 0.34 & 0.09 & 0.17 & 0.37 & 0.34 \\
\hline \multirow{3}{*}{$50 \times 5$} & 0 & 70 & 0 & 100 & 0 & 50 & 0 & 100 \\
\hline & 11.74 & 0.41 & 5.77 & 0 & 9.8 & 0.98 & 5.44 & 0 \\
\hline & 0.17 & 2.87 & 14.3 & 7.92 & 0.16 & 2.89 & 14.11 & 7.96 \\
\hline \multirow{3}{*}{$50 \times 10$} & 0 & 90 & 0 & 100 & 0 & 80 & 0 & 100 \\
\hline & 13.42 & 0.08 & 6.67 & 0 & 11.31 & 0.08 & 5.57 & 0 \\
\hline & 0.4 & 3.09 & 14.45 & 8.3 & 0.4 & 3.14 & 14.22 & 8.35 \\
\hline \multirow{3}{*}{$50 \times 20$} & 0 & 80 & 0 & 100 & 0 & 90 & 0 & 100 \\
\hline & 13.92 & 0.1 & 8.41 & 0 & 12.22 & 0.03 & 7.6 & 0 \\
\hline & 1.16 & 3.84 & 14.85 & 9.1 & 1.17 & 3.88 & 14.66 & 9.15 \\
\hline \multirow{3}{*}{ Average } & 0 & 80 & 0 & 100 & 0 & 73.33 & 0 & 100 \\
\hline & 13.03 & 0.2 & 6.95 & 0 & 11.11 & 0.37 & 6.2 & 0 \\
\hline & 0.58 & 3.27 & 14.53 & 8.44 & 0.57 & 3.3 & 14.33 & 8.49 \\
\hline \multirow{3}{*}{$100 \times 5$} & 0 & 70 & 0 & 100 & 0 & 60 & 0 & 100 \\
\hline & 10.11 & 0.73 & 6.08 & 0 & 8.27 & 0.36 & 5.32 & 0 \\
\hline & 0.83 & 40.7 & 226.25 & 119.23 & 0.67 & 40.75 & 225.78 & 121.01 \\
\hline \multirow{3}{*}{$100 \times 10$} & 0 & 40 & 0 & 100 & 0 & 30 & 0 & 100 \\
\hline & 11.16 & 0.85 & 7.07 & 0 & 8.68 & 0.43 & 6.68 & 0 \\
\hline & 1.67 & 39.97 & 226.09 & 118.98 & 1.69 & 40.17 & 226.26 & 118.94 \\
\hline \multirow{3}{*}{$100 \times 20$} & 0 & 60 & 0 & 100 & 0 & 80 & 0 & 100 \\
\hline & 11.42 & 0.26 & 7.54 & 0 & 9.81 & 0.14 & 6.74 & 0 \\
\hline & 5.6 & 43.73 & 225.7 & 122.87 & 5.07 & 43.82 & 226.89 & 123.65 \\
\hline \multirow{3}{*}{ Average } & 0 & 56.67 & 0 & 100 & 0 & 56.67 & 0 & 100 \\
\hline & 10.89 & 0.61 & 6.9 & 0 & 8.92 & 0.31 & 6.25 & 0 \\
\hline & 2.7 & 41.47 & 226.01 & 120.36 & 2.48 & 41.58 & 226.31 & 121.2 \\
\hline \multirow{3}{*}{$200 \times 10$} & 0 & 50 & 0 & 100 & 0 & 80 & 0 & 100 \\
\hline & 8.11 & 0.33 & 6.08 & 0 & 6.68 & 0.12 & 4.58 & 0 \\
\hline & 6.88 & 611.5 & 3799.28 & 1837.43 & 6.81 & 612.89 & 3759.58 & 1839.95 \\
\hline
\end{tabular}


Brazilian Journal of Operations \& Production Management Volume 12, Número 2, 2015, pp. 180-194 DOI: 10.14488/BJOPM.2015.v12.n2.a1

\begin{tabular}{ccccccccc}
\hline & 0 & 70 & 0 & 100 & 0 & 80 & 0 & 100 \\
$200 \times 20$ & 8.68 & 0.11 & 5.52 & 0 & 7.43 & 0.02 & 4.4 & 0 \\
& 21.63 & 626.84 & 3681.3 & 1834.39 & 20.81 & 629.81 & 3653.72 & 1843.07 \\
\hline \multirow{2}{*}{ Average } & 0 & 58.89 & 0 & 100 & 0 & 72.22 & 0 & 100 \\
& 9.23 & 0.35 & 6.16 & 0 & 7.68 & 0.15 & 5.07 & 0 \\
\hline * Success Rate (\%);** ARPD (\%);*** Average CPU time (second).
\end{tabular}

Table 2 - Comparison of results in Taillard's testbed SSD-100 and SSD-125 (Makespan)

\begin{tabular}{|c|c|c|c|c|c|c|c|c|}
\hline \multirow[b]{2}{*}{$n \times m$} & \multicolumn{4}{|c|}{ SSD-100 } & \multicolumn{4}{|c|}{ SSD-125 } \\
\hline & BAH & BIH & TRIPS & GAPH & ВАН & BIH & TRIPS & GAPH \\
\hline \multirow{3}{*}{$20 \times 5$} & 0 & 20 & 0 & 100 & 0 & 60 & 10 & 90 \\
\hline & 9.95 & 1.08 & 5.71 & 0 & 10.54 & 0.85 & 5.08 & 0.14 \\
\hline & 0.02 & 0.11 & 0.34 & 0.27 & 0.02 & 0.11 & 0.33 & 0.27 \\
\hline \multirow{3}{*}{$20 \times 10$} & 0 & 50 & 0 & 100 & 0 & 50 & 0 & 100 \\
\hline & 12.2 & 1.09 & 6.82 & 0 & 11.46 & 1.03 & 5.62 & 0 \\
\hline & 0.07 & 0.14 & 0.36 & 0.31 & 0.06 & 0.15 & 0.36 & 0.31 \\
\hline \multirow{3}{*}{$20 \times 20$} & 0 & 60 & 0 & 100 & 0 & 50 & 0 & 100 \\
\hline & 12.15 & 0.35 & 7.57 & 0 & 11.85 & 0.93 & 6.63 & 0 \\
\hline & 0.18 & 0.26 & 0.42 & 0.43 & 0.18 & 0.26 & 0.42 & 0.44 \\
\hline \multirow{3}{*}{ Average } & 0 & 43.33 & 0 & 100 & 0 & 53.33 & 3.33 & 96.67 \\
\hline & 11.44 & 0.84 & 6.7 & 0 & 11.28 & 0.94 & 5.78 & 0.05 \\
\hline & 0.09 & 0.17 & 0.37 & 0.34 & 0.09 & 0.17 & 0.37 & 0.34 \\
\hline \multirow{3}{*}{$50 \times 5$} & 0 & 50 & 0 & 100 & 0 & 30 & 0 & 100 \\
\hline & 9.51 & 0.8 & 4.38 & 0 & 9.21 & 1.32 & 5.7 & 0 \\
\hline & 0.17 & 2.88 & 14.11 & 8.01 & 0.17 & 2.88 & 14.11 & 8.02 \\
\hline \multirow{3}{*}{$50 \times 10$} & 0 & 70 & 0 & 100 & 0 & 60 & 0 & 100 \\
\hline & 8.98 & 0.13 & 4.01 & 0 & 9.97 & 0.93 & 4.99 & 0 \\
\hline & 0.39 & 3.12 & 14.27 & 8.37 & 0.4 & 3.11 & 14.3 & 8.34 \\
\hline \multirow{3}{*}{$50 \times 20$} & 0 & 80 & 0 & 100 & 0 & 90 & 0 & 100 \\
\hline & 9.95 & 0.04 & 5.53 & 0 & 9.07 & 0.01 & 5.44 & 0 \\
\hline & 1.19 & 3.85 & 14.71 & 9.13 & 1.16 & 3.85 & 14.74 & 9.13 \\
\hline \multirow{3}{*}{ Average } & 0 & 66.67 & 0 & 100 & 0 & 60 & 0 & 100 \\
\hline & 9.48 & 0.32 & 4.64 & 0 & 9.41 & 0.75 & 5.38 & 0 \\
\hline & 0.58 & 3.28 & 14.36 & 8.5 & 0.58 & 3.28 & 14.38 & 8.5 \\
\hline \multirow{3}{*}{$100 \times 5$} & 0 & 30 & 0 & 100 & 0 & 20 & 0 & 100 \\
\hline & 7.32 & 0.71 & 4.4 & 0 & 7.14 & 1.02 & 4.44 & 0 \\
\hline & 0.68 & 41.16 & 223.3 & 122.54 & 0.67 & 40.93 & 223.37 & 118.93 \\
\hline \multirow{3}{*}{$100 \times 10$} & 0 & 60 & 0 & 100 & 0 & 70 & 0 & 100 \\
\hline & 7.38 & 0.17 & 4.88 & 0 & 7.05 & 0.24 & 3.81 & 0 \\
\hline & 1.75 & 40.67 & 226.42 & 119.35 & 2.03 & 40.36 & 227.38 & 119.32 \\
\hline
\end{tabular}


Brazilian Journal of Operations \& Production Management

Volume 12, Número 2, 2015, pp. 180-194

DOI: 10.14488/BJOPM.2015.v12.n2.a1

\begin{tabular}{ccccccccc}
\hline & 0 & 70 & 0 & 100 & 0 & 90 & 0 & 100 \\
$100 \times 20$ & 8.05 & 0.37 & 5.37 & 0 & 6.82 & 0.08 & 4.47 & 0 \\
& 5.18 & 43.87 & 228.77 & 123.35 & 5.19 & 44.09 & 229.54 & 123.4 \\
\hline \multirow{2}{*}{ Average } & 0 & 53.33 & 0 & 100 & 0 & 60 & 0 & 100 \\
& 7.58 & 0.42 & 4.89 & 0 & 7 & 0.44 & 4.24 & 0 \\
& 2.54 & 41.9 & 226.16 & 121.75 & 2.63 & 41.8 & 226.76 & 120.55 \\
\hline \multirow{2}{*}{$200 \times 10$} & 0 & 40 & 0 & 100 & 0 & 30 & 0 & 100 \\
& 6.37 & 0.59 & 4.21 & 0 & 5.59 & 0.56 & 3.44 & 0 \\
& 6.87 & 613.21 & 3780.1 & 1853.66 & 7.03 & 618 & 3793.94 & 1836.08 \\
\hline \multirow{2}{*}{$200 \times 20$} & 0 & 60 & 0 & 100 & 0 & 60 & 0 & 100 \\
& 6.55 & 0.09 & 3.45 & 0 & 5.7 & 0.09 & 3.15 & 0 \\
& 21.19 & 632.35 & 3657.28 & 1846.33 & 21.13 & 633.6 & 3649.61 & 1844.22 \\
\hline & 0 & 51.11 & 0 & 100 & 0 & 50 & 0 & 100 \\
& 0.83 & 0.36 & 4.18 & 0 & 6.1 & 0.36 & 3.61 & 0 \\
& 6.83 & 424.95 & 2554.51 & 1273.91 & 10.26 & 424.02 & 2556.77 & 1266.95 \\
\hline
\end{tabular}

One interesting characteristics from the experimental analysis is that the methods seem to be unaffected by distribution of processing or setup times, i.e., there are no better methods depending on the specific distribution of processing or setup times.

Comparing GAPH with $\mathrm{BIH}$, we observe that GAPH always gets equal or better results than $\mathrm{BIH}$. The minimum success rate of GAPH was $90 \%$, while the minimum of BIH was $20 \%$.

With respect to the CPU time, TRIPS require much more computational effort than GAPH and $\mathrm{BIH}$. As it can be observed in Tables 1 and 2, for the biggest problem analyzed (200x20), the average CPU time of TRIPS was nearly 3660s, while TRIPS required nearly 630s and GAPH required nearly 1850s.

Finally, our proposal heuristic is statistically better than the rest of the heuristics, although it is more time consuming than BIH. GAPH always gets the better result, and is more efficient than TRIPS.

Table 3 - Comparison of results in Taillard's testbed SSD-10 and SSD-50 (Total Flowtime)

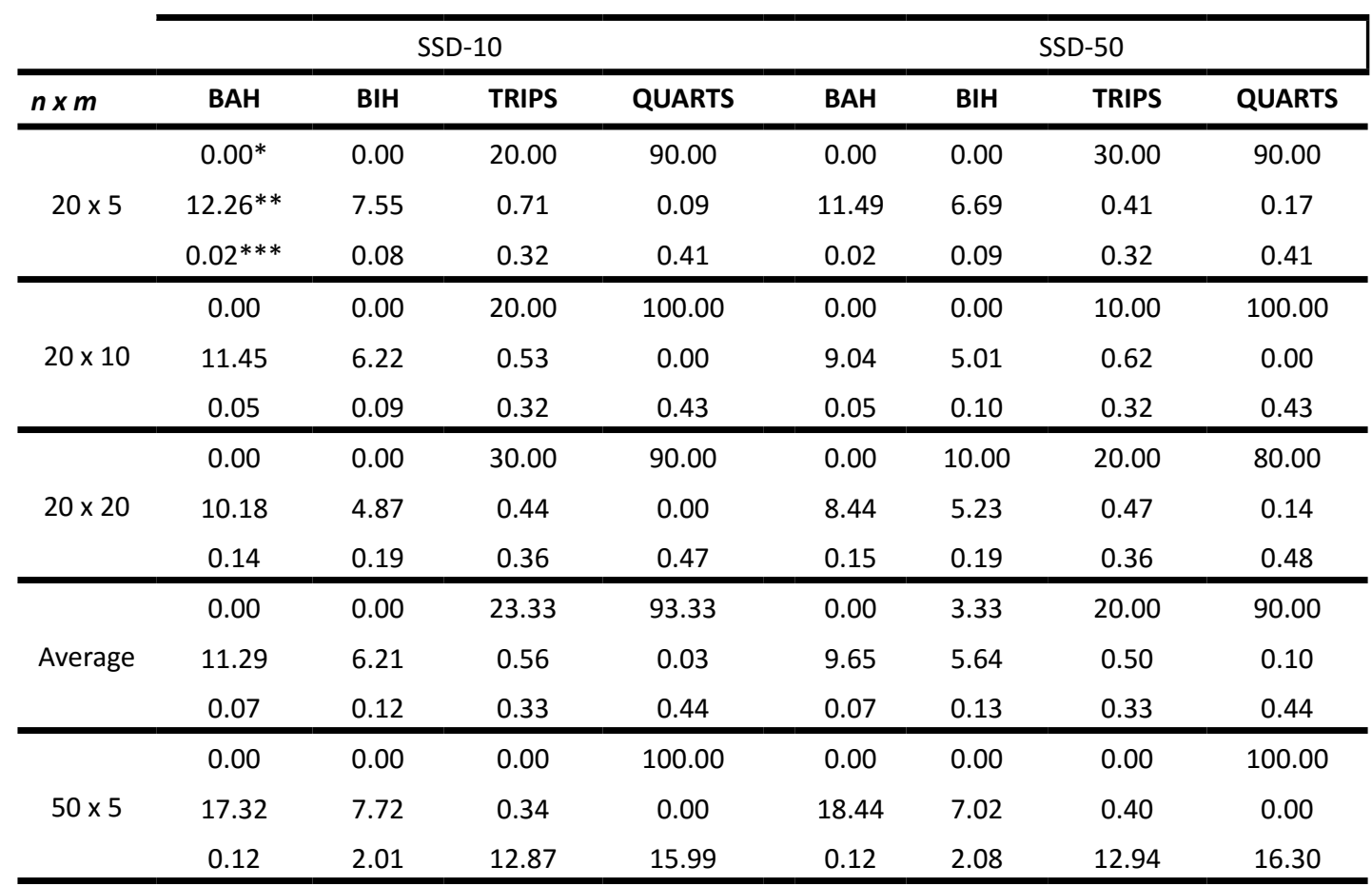




\begin{tabular}{|c|c|c|c|c|c|c|c|c|}
\hline & 0.00 & 0.00 & 0.00 & 100.00 & 0.00 & 0.00 & 20.00 & 80.00 \\
\hline \multirow[t]{3}{*}{$50 \times 10$} & 20.24 & 7.90 & 0.39 & 0.00 & 17.64 & 6.90 & 0.24 & 0.11 \\
\hline & 0.31 & 2.35 & 13.00 & 16.65 & 0.31 & 2.27 & 13.07 & 16.75 \\
\hline & 0.00 & 0.00 & 0.00 & 100.00 & 0.00 & 0.00 & 0.00 & 100.00 \\
\hline \multirow[t]{3}{*}{$50 \times 20$} & 18.51 & 6.95 & 0.68 & 0.00 & 17.08 & 6.07 & 0.64 & 0.00 \\
\hline & 0.92 & 2.86 & 13.98 & 16.91 & 0.91 & 2.84 & 14.27 & 17.16 \\
\hline & 0.00 & 0.00 & 0.00 & 100.00 & 0.00 & 0.00 & 6.67 & 93.33 \\
\hline \multirow[t]{3}{*}{ Average } & 18.69 & 7.52 & 0.47 & 0.00 & 17.72 & 6.66 & 0.43 & 0.04 \\
\hline & 0.45 & 2.41 & 13.28 & 16.52 & 0.45 & 2.40 & 13.43 & 16.74 \\
\hline & 0.00 & 0.00 & 0.00 & 100.00 & 0.00 & 0.00 & 0.00 & 100.00 \\
\hline \multirow[t]{3}{*}{$100 \times 5$} & 22.93 & 8.11 & 0.27 & 0.00 & 24.94 & 6.85 & 0.17 & 0.00 \\
\hline & 0.50 & 32.12 & 217.34 & 258.54 & 0.51 & 32.78 & 215.95 & 259.79 \\
\hline & 0.00 & 0.00 & 0.00 & 100.00 & 0.00 & 0.00 & 0.00 & 100.00 \\
\hline \multirow[t]{3}{*}{$100 \times 10$} & 24.09 & 7.20 & 0.52 & 0.00 & 21.25 & 6.14 & 0.46 & 0.00 \\
\hline & 1.25 & 33.01 & 216.13 & 265.60 & 1.26 & 32.48 & 216.72 & 265.86 \\
\hline & 0.00 & 0.00 & 10.00 & 90.00 & 0.00 & 0.00 & 10.00 & 90.00 \\
\hline \multirow[t]{3}{*}{$100 \times 20$} & 25.07 & 6.06 & 0.36 & 0.21 & 20.88 & 5.07 & 0.26 & 0.04 \\
\hline & 3.70 & 35.35 & 218.32 & 265.61 & 3.72 & 35.70 & 218.47 & 268.15 \\
\hline & 0.00 & 0.00 & 3.33 & 96.67 & 0.00 & 0.00 & 3.33 & 96.67 \\
\hline \multirow[t]{3}{*}{ Average } & 24.03 & 7.12 & 0.39 & 0.07 & 22.36 & 6.02 & 0.30 & 0.01 \\
\hline & 1.82 & 33.49 & 217.26 & 263.25 & 1.83 & 33.65 & 217.05 & 264.60 \\
\hline & 0.00 & 0.00 & 0.00 & 100.00 & 0.00 & 0.00 & 0.00 & 100.00 \\
\hline \multirow[t]{3}{*}{$200 \times 10$} & 28.80 & 6.27 & 0.21 & 0.00 & 26.12 & 5.47 & 0.34 & 0.00 \\
\hline & 5.15 & 511.98 & 3494.11 & 4133.52 & 5.17 & 513.02 & 3490.31 & 4130.08 \\
\hline & 0.00 & 0.00 & 10.00 & 90.00 & 0.00 & 0.00 & 0.00 & 100.00 \\
\hline \multirow[t]{3}{*}{$200 \times 20$} & 31.86 & 6.04 & 0.13 & 0.04 & 27.83 & 5.83 & 0.09 & 0.00 \\
\hline & 14.90 & 515.71 & 3537.08 & 4164.64 & 14.82 & 515.82 & 3517.09 & 4158.26 \\
\hline & 0.00 & 0.00 & 5.00 & 95.00 & 0.00 & 0.00 & 0.00 & 100.00 \\
\hline \multirow[t]{2}{*}{ Average } & 30.33 & 6.16 & 0.17 & 0.02 & 26.97 & 5.65 & 0.22 & 0.00 \\
\hline & 10.03 & 513.85 & 3515.60 & 4149.08 & 10.00 & 514.42 & 3503.70 & 4144.17 \\
\hline
\end{tabular}

Table 4 - Comparison of results in Taillard's testbed SSD-100 and SSD-125 (Total Flowtime)

\begin{tabular}{ccccccccc} 
& \multicolumn{3}{c}{ SSD-100 } & \multicolumn{5}{c}{ SSD-125 } \\
\hline $\boldsymbol{n} \boldsymbol{x} \boldsymbol{x} \boldsymbol{m}$ & BAH & BIH & TRIPS & QUARTS & BAH & BIH & TRIPS & QUARTS \\
\hline \multirow{2}{*}{$20 \times 5$} & 0.00 & 0.00 & 30.00 & 100.00 & 0.00 & 0.00 & 70.00 & 100.00 \\
& 15.47 & 5.21 & 0.38 & 0.00 & 17.89 & 7.11 & 0.29 & 0.00 \\
& 0.02 & 0.08 & 0.32 & 0.42 & 0.02 & 0.09 & 0.32 & 0.43 \\
\hline \multirow{2}{*}{$20 \times 10$} & 0.00 & 0.00 & 30.00 & 90.00 & 0.00 & 0.00 & 30.00 & 90.00 \\
& 10.57 & 5.79 & 0.55 & 0.02 & 11.05 & 5.80 & 0.34 & 0.20 \\
& 0.05 & 0.09 & 0.33 & 0.43 & 0.05 & 0.09 & 0.32 & 0.44 \\
\hline
\end{tabular}


Brazilian Journal of Operations \& Production Management

Volume 12, Número 2, 2015, pp. 180-194

DOI: 10.14488/BJOPM.2015.v12.n2.a1

\begin{tabular}{|c|c|c|c|c|c|c|c|c|}
\hline & 0.00 & 0.00 & 40.00 & 90.00 & 0.00 & 0.00 & 10.00 & 100.00 \\
\hline \multirow[t]{3}{*}{$20 \times 20$} & 8.57 & 4.66 & 0.34 & 0.00 & 8.96 & 4.54 & 0.32 & 0.00 \\
\hline & 0.14 & 0.20 & 0.36 & 0.47 & 0.14 & 0.20 & 0.36 & 0.48 \\
\hline & 0.00 & 0.00 & 33.33 & 93.33 & 0.00 & 0.00 & 36.67 & 96.67 \\
\hline \multirow[t]{3}{*}{ Average } & 11.54 & 5.22 & 0.43 & 0.01 & 12.63 & 5.82 & 0.32 & 0.07 \\
\hline & 0.07 & 0.12 & 0.34 & 0.44 & 0.07 & 0.13 & 0.33 & 0.45 \\
\hline & 0.00 & 0.00 & 10.00 & 100.00 & 0.00 & 0.00 & 60.00 & 70.00 \\
\hline \multirow[t]{3}{*}{$50 \times 5$} & 25.77 & 7.76 & 0.29 & 0.00 & 27.32 & 8.15 & 0.24 & 0.46 \\
\hline & 0.12 & 2.04 & 12.97 & 16.67 & 0.13 & 2.05 & 12.96 & 16.55 \\
\hline & 0.00 & 0.00 & 0.00 & 100.00 & 0.00 & 0.00 & 0.00 & 100.00 \\
\hline \multirow[t]{3}{*}{$50 \times 10$} & 19.1 & 6.58 & 0.24 & 0.00 & 19.69 & 6.80 & 0.30 & 0.00 \\
\hline & 0.31 & 2.29 & 13.11 & 16.82 & 0.31 & 2.22 & 13.10 & 16.93 \\
\hline & 0.00 & 0.00 & 0.00 & 100.00 & 0.00 & 0.00 & 0.00 & 100.00 \\
\hline \multirow[t]{3}{*}{$50 \times 20$} & 16.28 & 4.78 & 0.30 & 0.00 & 15.37 & 4.83 & 0.24 & 0.00 \\
\hline & 0.92 & 2.87 & 14.26 & 17.25 & 0.92 & 2.85 & 14.10 & 17.47 \\
\hline & 0.00 & 0.00 & 3.33 & 100.00 & 0.00 & 0.00 & 20.00 & 90.00 \\
\hline \multirow[t]{3}{*}{ Average } & 20.38 & 6.37 & 0.28 & 0.00 & 20.79 & 6.59 & 0.26 & 0.15 \\
\hline & 0.45 & 2.40 & 13.45 & 16.91 & 0.45 & 2.37 & 13.39 & 16.98 \\
\hline & 0.00 & 0.00 & 0.00 & 100.00 & 0.00 & 0.00 & 30.00 & 100.00 \\
\hline \multirow[t]{3}{*}{$100 \times 5$} & 30.5 & 6.37 & 0.13 & 0.00 & 33.78 & 6.68 & 0.05 & 0.00 \\
\hline & 0.50 & 33.21 & 215.57 & 265.68 & 0.51 & 32.97 & 218.75 & 267.20 \\
\hline & 0.00 & 0.00 & 0.00 & 100.00 & 0.00 & 0.00 & 10.00 & 90.00 \\
\hline \multirow[t]{3}{*}{$100 \times 10$} & 23.51 & 5.69 & 0.16 & 0.00 & 23.67 & 6.08 & 0.12 & 0.06 \\
\hline & 1.27 & 32.70 & 217.09 & 269.60 & 1.27 & 32.78 & 217.73 & 271.06 \\
\hline & 0.00 & 0.00 & 0.00 & 100.00 & 0.00 & 0.00 & 0.00 & 100.00 \\
\hline \multirow[t]{3}{*}{$100 \times 20$} & 19.67 & 4.71 & 0.22 & 0.00 & 18.91 & 4.27 & 0.16 & 0.00 \\
\hline & 3.71 & 34.97 & 219.34 & 269.38 & 3.72 & 35.02 & 218.98 & 270.50 \\
\hline & 0.00 & 0.00 & 0.00 & 100.00 & 0.00 & 0.00 & 13.33 & 96.67 \\
\hline \multirow[t]{3}{*}{ Average } & 24.56 & 5.59 & 0.17 & 0.00 & 25.45 & 5.68 & 0.11 & 0.02 \\
\hline & 1.83 & 33.63 & 217.33 & 268.22 & 1.83 & 33.59 & 218.48 & 269.58 \\
\hline & 0.00 & 0.00 & 20.00 & 80.00 & 0.00 & 0.00 & 0.00 & 100.00 \\
\hline \multirow[t]{3}{*}{$200 \times 10$} & 27.88 & 5.17 & 0.07 & 0.04 & 28.71 & 5.69 & 0.05 & 0.00 \\
\hline & 5.18 & 511.11 & 3502.32 & 4158.17 & 5.15 & 512.84 & 3522.19 & 4165.47 \\
\hline & 0.00 & 0.00 & 0.00 & 100.00 & 0.00 & 0.00 & 20.00 & 80.00 \\
\hline \multirow[t]{3}{*}{$200 \times 20$} & 24.30 & 5.27 & 0.09 & 0.00 & 24.30 & 5.27 & 0.09 & 0.00 \\
\hline & 14.91 & 514.98 & 3528.10 & 4166.21 & 14.87 & 515.03 & 3518.97 & 4178.01 \\
\hline & 0.00 & 0.00 & 10.00 & 90.00 & 0.00 & 0.00 & 10.00 & 90.00 \\
\hline \multirow[t]{2}{*}{ Average } & 26.09 & 5.22 & 0.08 & 0.02 & 26.50 & 5.48 & 0.07 & 0.00 \\
\hline & 10.05 & 513.05 & 3515.21 & 4162.19 & 10.01 & 513.94 & 3520.58 & 4171.74 \\
\hline
\end{tabular}


As can be seen from the results in Tables 3 and 4, QUARTS heuristic obtains better results than the rest of the constructive heuristics. The success rate of QUARTS is superior to all heuristics, in all problems tested. QUARTS obtained the best solution in $95.23 \%$ of all problems tested, while TRIPS only obtained the best solution in $12.27 \%$ of them. We observed that TRIPS success rate decays with the increase of the number of jobs, indicating that this method works better for small problems. BAH and $\mathrm{BIH}$ are not competitive with the other heuristics tested. All success rates for $\mathrm{BAH}$ were zero, and $\mathrm{BIH}$ only got the best solution once.

Considering the ARPD, BAH has the highest values. Its ARPD exceeds $30 \%$ in some cases. The second method with the highest ARPD is $\mathrm{BIH}$, with an ARPD of $5 \%$ in average. Analyzing TRIPS, we can see that its higher ARPD is $0.71 \%$, and its ARPD average is $0.31 \%$. Despite TRIPS success rate decays with the increase of jobs, its ARPD shows that the method gets better results with the increase of jobs. Its ARPD decays with the increase of jobs. The ARPD analysis ratifies the superiority of QUARTS, regarding the solution quality. Its ARPD average is $0.04 \%$, about 8 times lower than TRIPS.

With respect to the CPU time, $\mathrm{BAH}$ is the fastest method, followed by BIH. The average CPU time of BAH was about $2.5 \mathrm{~s}$, while BIH required nearly 104s. Comparing TRIPS and QUARTS, we can see that TRIPS is a little faster than QUARTS. The average CPU time of TRIPS was about 702s, while QUARTS required 833s. As we can see in Tables 3 and 4 , the CPU time of both methods always have the same order of precision. So, the difference between them is not significant.

Finally, among the four methods evaluated in this computational experiment for the total flow time criteria, we can see that QUARTS is the better one, regarding the solution quality. In average, its success rate is $95.10 \%$ and its ARPD is $0.04 \%$, while the methods $\mathrm{BAH}, \mathrm{BIH}$ and TRIPS have success rates of $0 \%, 0.23 \%$ and $12.27 \%$ and ARPDs of $19.93 \%, 6.10 \%$ and $0.31 \%$, respectively. With respect to the CPU time, although TRIPS is a little bit faster than QUARTS, this difference is insignificant. Thus, we can conclude that QUARTS is the better constructive heuristic for the total flow time criteria.

\section{CONCLUSION}

In this paper, we dealt with the problem of scheduling a no-wait flow shop with sequence-dependent setup times with both make span and total flow time objectives by means of constructive heuristics. We presented two new heuristics, named GAPH and QUARTS, for the make span and total flow time criteria, respectively, and carried out extensive computational experiment. The results showed that GAPH and QUARTS get better results than the other constructive heuristics tested. Henceforth, it can be concluded that the proposed heuristics obtain high solution quality comparing to the existing constructive heuristics for the problems, in acceptable computational times.

\section{ACKNOWLEDGMENT}

The research of the first author is partially supported by The State of São Paulo Research Foundation (FAPESP) under grant number 09/06832-2. The research of the second author is partially supported by a grant number 473654/2009-1 from the National Council for Scientific and Technological Development (CNPq), Brazil.

\section{REFERENCES}

Aldowaisan, T. (2001). A new heuristic and dominance relations for no-wait flowshops with setups, Computers \& Operations Research, 28 (6), 563-584.

Allahverdi, A. \& Aldowaisan, T. (2001). Minimizing total completion time in a no-wait flowshop with sequencedependent additive changeover times, Journal of the Operational Research Society, 52 (4), 449-462.

Allahverdi, A., Ng, C.T., Cheng, T.C.E. \& Kovalyov, M.Y. (2008). A survey of scheduling problems with setups times or costs, European Journal of Operational Research, 187 (3), 985-1032.

Allahverdi, A. \& Soroush, H.M. (2008). The significance of reducing setup times/setup costs, European Journal of Operational Research, 187 (3), 978-984.

Bianco, L., Dell'Olmo, P. \& Giordani, S. (1999). Flow shop no-wait scheduling with sequence-dependent setup times and release dates, INFOR Journal, 37 (1), 3-19.

Brown, S.I., Mcgarvey, R. \& Ventura, J.A. (2004). Total flowtime and makespan for a no-wait $m$-machine flowshop with set-up times separated, Journal of the Operational Research Society, 55 (6), 614-621.

França, P.M., Tin Jr, G. \& Buriol, L.S. (2006). Genetic Algorithms for the no-wait flowshop sequencing problem with time restrictions, International Journal of Production Research, 44 (5), 939-957.

Gupta, J.N.D. (1986). Flowshop schedules with sequencedependent setup times, Journal of Operations Research Society of Japan, 29 (3), 206-219.

Ruiz, R., Maroto, C. \& Alcaraz, J. (2005). Solving the flowshop scheduling problem with sequence-dependent setup times using advanced metaheuristics, European Journal of Operational Research, 165 (1), 34-54. 
Stafford Jr, E.F. \& Tseng, F.T. (1990). On the SrikarGhosh MILP model for the NxM SDST flowshop problem, International Journal of Production Research, 28 (10), 18171830.

Stafford Jr, E.F. \& Tseng, F.T. (2002). Two models for a family of flowshop sequencing problems, European Journal of Operational Research, 142 (2), 282-293.

Taillard, E. (1993). Benchmarks for basic scheduling problems, European Journal of Operational Research, 64 (2), 278-285. 